Review

\title{
Characterization of the Asian Phenotype - An Emerging Paradigm with Clinicopathological and Human Research Implications
}

\author{
Melvin Khee-Shing Leow ${ }^{\bowtie}$ \\ Clinical Investigator, Singapore Institute for Clinical Sciences, A*STAR; Deputy Director, Clinical Nutrition Research Centre (CNRC), Singapore; Senior \\ Consultant Endocrinologist, Tan Tock Seng Hospital; Clinician Scientist, National Healthcare Group (NHG); Clinical Associate Professor, National University of \\ Singapore; Adjunct Associate Professor, Duke-NUS Graduate Medical School, Singapore; Associate Professor, Lee Kong Chian School of Medicine, Nanyang \\ Technological University, Singapore. \\ $\triangle$ Corresponding author: Melvin Khee-Shing Leow, MBBS, MSc, MMed (Int Med), PhD, FAMS, FACE, FACP, FRCP(Edin), melvin_leow@nuhs.edu.sg \\ (C) Ivyspring International Publisher. This is an open access article distributed under the terms of the Creative Commons Attribution (CC BY-NC) license \\ (https://creativecommons.org/licenses/by-nc/4.0/). See http://ivyspring.com/terms for full terms and conditions.
}

Received: 2016.12.22; Accepted: 2017.03.14; Published: 2017.06.15

\begin{abstract}
Background: Modern medicine recognizes that salient, inherent variations between Caucasians and Asians exist. Radical changes are occurring in the health scene with increasing emphasis centered on the recognition of inter-individual variations unique to Asians that impact on medical management and outcomes.

Aim: This review analyzes distinct features or outcomes in terms of epidemiology, disease thresholds, diagnostic cutoffs and treatment responses of Asian people compared with non-Asians.

Methods: This review is based on a literature search via PubMed and MEDLINE for relevant articles related to the Asian phenotype and its impact on health and disease.

Results: An 'Asian phenotype' could be characterized across the spectrum of biomedical disciplines and underscores the major challenges clinicians must face in their daily management of a cosmopolitan population and their extrapolation of research outcomes.

Conclusion: Interventions for various ailments that have traditionally ignored population differences have now entered the age of personalized, stratified or precision medicine requiring an individualized approach being adopted as a new standard of care. Factoring in Asian phenotypes is essential for the medical research community and the development of improved clinical practice guidelines across a continuum of disciplines that will ultimately translate to better human health round the world.
\end{abstract}

Key words: Asian phenotype, Personalized medicine, Diagnostic cutoffs, Ethnicity, Inter-individual variations.

\section{Introduction}

With respect to both health and disease, increasing attention is being paid to the differences observed between the Caucasians of the West and the Asians of the East. Although the overall physiologic characteristics and biochemical makeup are virtually similar between the different races and ethnicities among human beings from various geographical locations despite overt differences in terms of anatomical and anthropometric parameters such as height and overall body frame, an emerging body of evidence suggests that certain significant and unique features documented in a range of conditions do appear to separate Asians from the rest of the world thus leading to the popularised conjecture of an "Asian phenotype". This is applicable not only to the pharmaceutical industries, but equally to academics, scientists and physicians practicing at the bedside.

In recent times, the Asian phenotype has claimed centre stage within the pharmaceutical industry, leading to the replication of many studies and clinical 
trials conducted on predominantly non-Asians subjects in their attempts to elucidate any major differences in drug metabolism and toxicity when translated to an Asian population. Until very recently, Japanese regulatory authorities represented one such extreme example by adopting a very stringent approach that disallowed the use of foreign clinical data for domestic product approval. Any drug or medical product/device registered overseas still has to be re-evaluated through repeating clinical studies by their pharmaceutical industries on Japanese subjects before these products can be approved and registered for use in their population [1].

\section{Method}

Articles on the subject area related to the Asian phenotype published in the extant literature up until November 2016 were searched using PubMed and Medline by exploding and combining the medical subject heading terms 'Asian continental ancestry group' or 'Asian [all fields]' and 'continental [all fields]' and 'ancestry [all fields]' and 'phenotype [all fields]' and using free-text words 'Asian phenotype', 'ethnic differences' or 'racial differences'. Searches were limited to full-text English-language papers. Publications discussing on health and disease-specific conditions in which evidence for the existence of an Asian phenotype was unequivocal were selected for review.

\section{The Asian Peoples of the World}

\section{Asian People}

Asians are in general considered to be people who are native inhabitants of Asia, and often loosely associated with people originating from East Asia like China, Japan, Korea.' In the broadest sense, it refers to all people from the Asia continent. Asia itself is the world's largest continent holding over four billion people, or roughly above $60 \%$ of the current human population [2].

\section{Varying Physical Attributes and Diagnostic Cutoffs}

Caucasian refers to the 'white race' of Mankind derived from the region of the Caucasus Mountains in Europe. Presently, the United States National Library of Medicine has discontinued this racial term as antiquated in favor of the term 'European' [3]. For the sake of this discussion, we will adopt the term Westerners to encompass the Europeans and the white race of the United Kingdom and North America.

To begin with, certain physical attributes distinguish Asians from Westerners. Asians generally tend to be smaller in body frame, based on the wrist circumference or elbow width, compared with Westerners when controlled for the same age and gender $[4,5]$. Complementing observations of overt differences in terms of physique, studies have confirmed that the average height and weight of the Asian population are generally lower than their Westerners counterparts [6, 7]. The health implications of these differences are crucial because many disease thresholds of obesity-related illnesses are significantly different when Asians are compared to other populations for a given range of anthropometric indices when adjusted for age and sex.

\section{Epidemiology and Definition of the Asian Phenotype}

\section{Asian-Prevalent Diseases}

Diseases which are 'quantitatively' commoner among Asians serve as a start-point to advance the Asian phenotype concept. Asian-prevalent diseases are those with a high disease burden and exhibiting differences in prevalence in Asia relative to the Western countries. Epidemiological data from healthcare organizations and disease registries reveal insights into etiology and disease biology, genetic predilection or uniqueness of those diseases in Asians. Knowledge surrounding Asian-prevalent diseases guides research investment and aligns public health policies. A number of conditions, such as nasopharyngeal carcinoma, Brugada syndrome and thyrotoxic periodic paralysis strikingly affect Asians compared to non-Asians.

\section{The Asian Phenotype}

Recognition of 'qualitative differences' in the expression of diseases between Asians and non-Asians is the key definition of the Asian phenotype which probably exists across a continuum of health and disease (Figure 1), thereby making it crucial to investigate its possible origins and implications it has for science and medicine. Nearly $85 \%$ of every human variant alleles can be found in any urban average population worldwide; about $6 \%$ of variability occurs among populations from the same continent, and some $9-13 \%$ of genetic variation is found between those from different continents [8]. However, numerous studies of drug-metabolizing enzymes generally show no more than 2- to 3-fold differences between races or ethnic groups as opposed to the often 10- to greater than 40-fold differences between individuals within any one race or ethnic group [9]. 


\section{Hypothesis of the Origin of the Asian Phenotype}

\section{Origin and Spread of Modern Humans}

Our human history could hold the key explanation of the origin of the Asian phenotype. Asian peoples as a human species may exhibit unique phenotypic features inherently based on their underlying origin. Paleontology, population genetics and molecular biology have served evolutionary biologists by informing the nature of human origin and global human genome diversity. Mitochondrial DNA sequences, Y-chromosome studies, single nucleotide polymorphisms (SNPs), insertions/deletions and microsatellites analyses thus far support the Displacement Theory that modern humans originated in Africa about 200,000 years ago [10] and then spread across to the Middle East, Asia, Australia, and Europe 60,000 to 70,000 years ago [11].

Asians represent the oldest out-of-Africa exodus, and Caucasians of Europe appeared temporally later about 35,000 years ago (Figure 2). Cladistic dendrograms computed on phylogenetic data provide useful insights on Asian divergence from a common ancestor and give clues to the phenotypic variations distinctive of this major group of Homo sapiens sapiens (Figure 3) [12]. Different epochs of peopling the planet probably explained the clustering of gene pools adapted to unique exposomes within Asia for robust survival.

\section{Molecular Genomics}

The Human Genome Project had initially revealed that Homo sapiens sapiens are genomically more uniform than expected, with only trivial DNA sequence divergence in transcription factor, signal transduction, receptor or biochemical transporter genes. Presently, it is thought that studies using SNPs have understated the extent of inter-human genetic variations. Copy number variations (CNV), inversions and other large structural variations are currently being examined to explain the observed differences between Asians and Westerners [13].

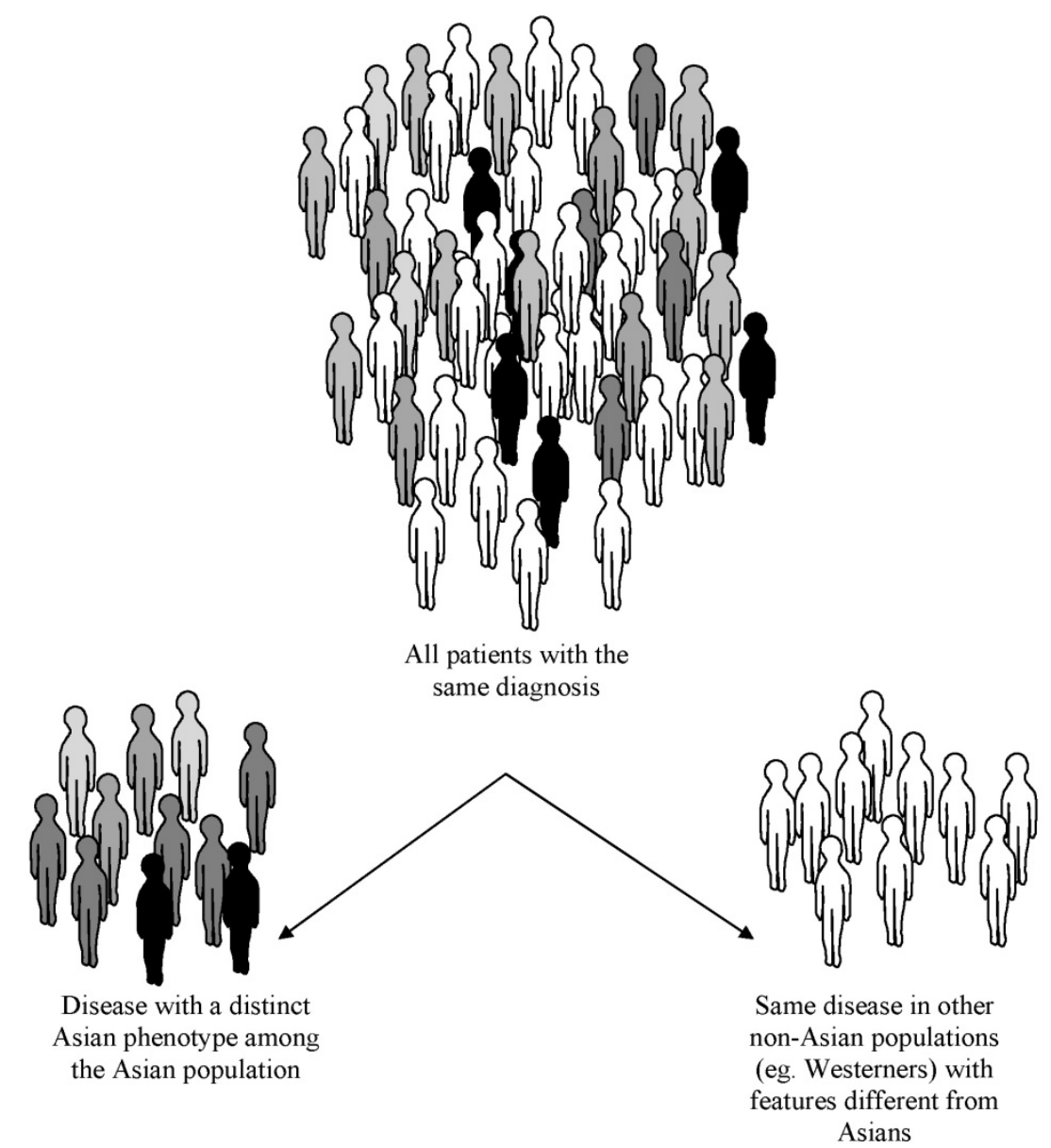

Figure 1. Concept of the Asian phenotype. In this schematic, a disease may occur in any human population round the world. However, the same medical condition may have unique features shared mainly by Asians but not in Westerners. 


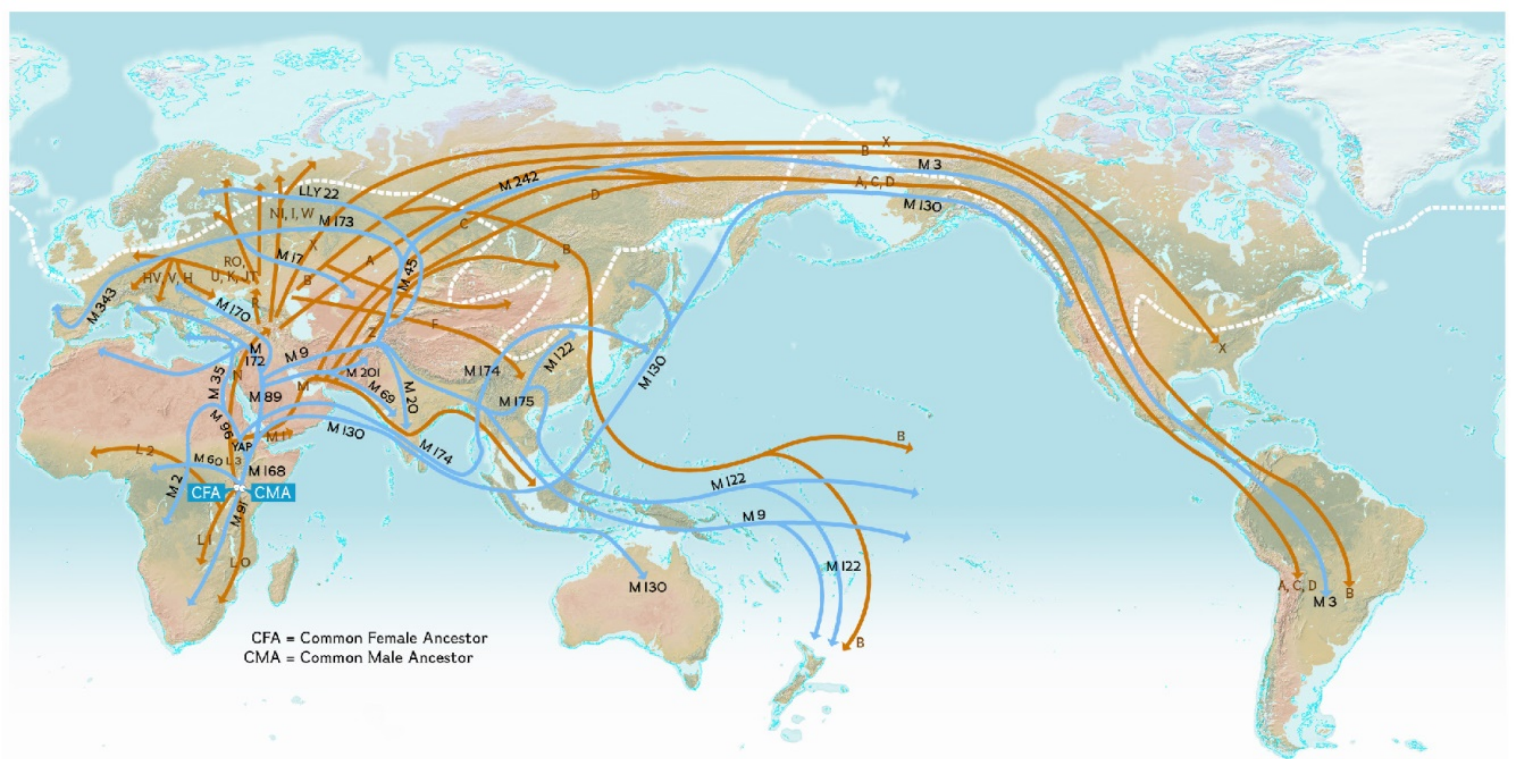

Figure 2. World map depicting the origin and migration of early modern humans. Early humans migrated from an 'epicenter' beginning about 100,000 years ago in the "Out-of-Africa" Theory and spread across the globe via routes based on DNA evidence using mitochondrial (orange) and Y-chromosome (blue) DNA. (Figure reproduced with permission from the National Geographic Society)

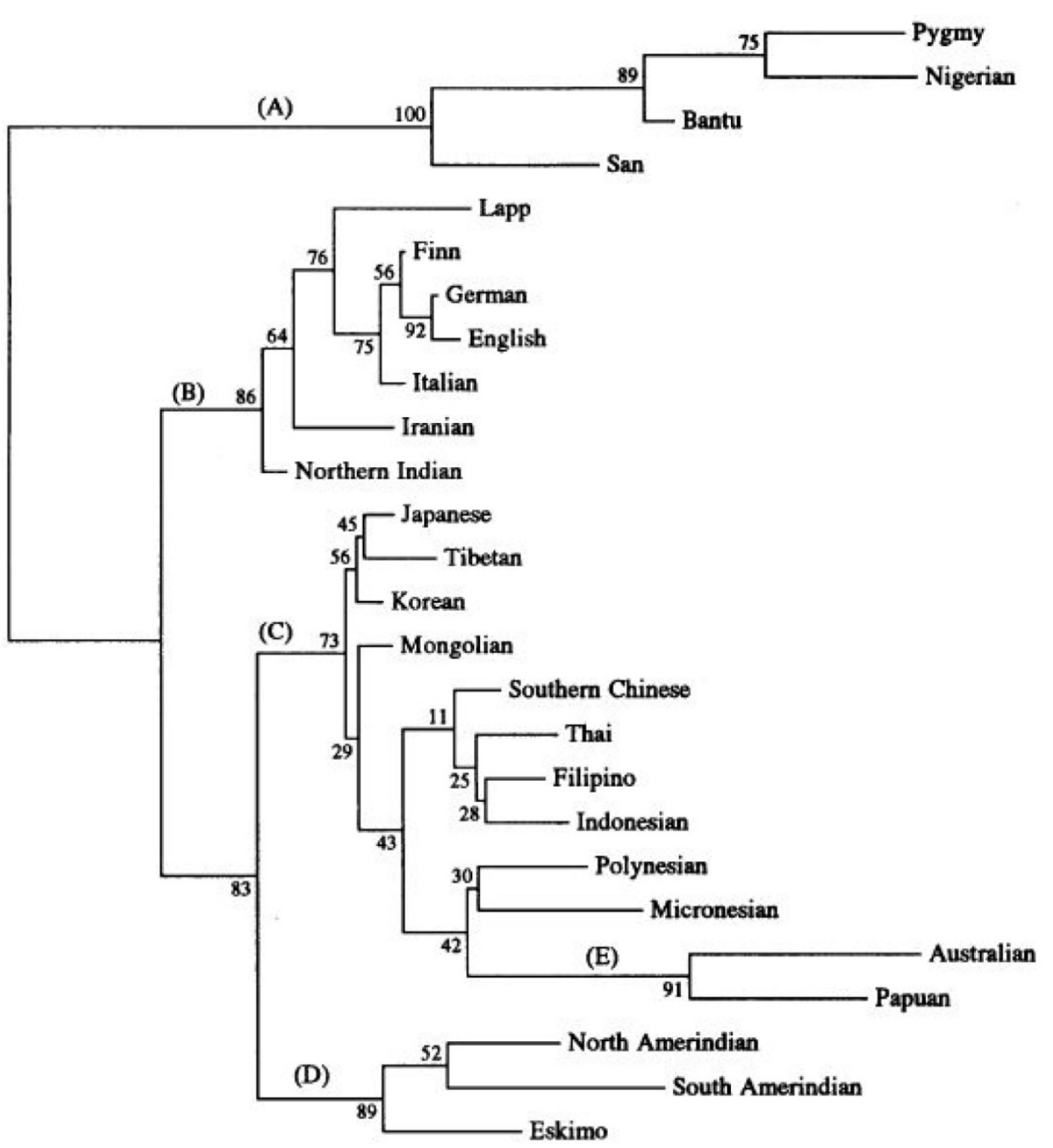

Figure 3. Phylogenetic cladogram of 26 human populations (from Nei and Roychoudhury, 1993) showing the major evolutionary divisions Africans (A), Caucasians (B), Greater Asians (C), Amerindians (D) and Australopapuans (E). Cladistic dendrograms computed on phylogenetic data yield insights on divergence of homo sapiens from a common ancestor and lend support to phenotypic variations distinctive of human populations in the continent defined as Asians. (Figure reproduced with permission from Oxford University Press) 


\section{Phenotype Plasticity}

Early humans dwelling in Asia could have acquired distinctive traits and conserved specific ranges of responses in health and disease governed by the rules of phenotype plasticity amidst complex environmental cues peculiar to the Asia continent over the course of millenia (Figure 4). Phenotype plasticity is the degree to which an individual's phenotype is shaped by its genotype "predicting" and responding to the environment through adaptive evolution dictated by the forces of natural selection. Alleles at various chromosomal loci function in a plastic response code to generate molecules with specific kinetic profiles that influence distinct developmental pathways. At the heart of the nature-nurture interaction, environment-dependent regulatory switches sense environmental cues and in turn elicit a switch between alternative developmental pathways that determine the overall fate of the final phenotype optimally adapted for that environment [14], mediated by pleiotropy and/or epistasis.

\section{Disease Presentation with unique features indicative of an Asian Phenotype}

\section{Endocrinology and Metabolism}

\section{Obesity and Type 2 Diabetes Mellitus}

There is an excess of type 2 diabetes mellitus among urban and migrant Asian Indians above and beyond what is purely predicted by traditional risk factors alone. It is now recognized that abdominal adiposity varies between Westerners and Asians and that cutoffs originally proposed in diagnostic guidelines for metabolic syndrome (eg. NCEP ATP III) tend to underestimate Asians [15]. Asian Indians and East Asians have smaller body size, more truncal and abdominal adiposity but lower average waist circumference than Westerners [16, 17]. Average waist/hip circumference ratios are higher in South Asians than in Europeans of similar BMI. Cross-sectional data from the 1998 Singapore National Health Survey involving 4,723 men and women of Chinese, Malay, and Asian-Indian ethnicity aged 18-69 years generated receiver operating characteristic analysis that suggested a waist circumference $>80 \mathrm{~cm}$ in women and $>90 \mathrm{~cm}$ in men was a more appropriate definition of central obesity in this population. Notably, South Asian neonates born in the Netherlands revealed a thin-fat insulin resistant phenotype compared to a matched Dutch white controls group [18]. Asians, as such, appear to have a distinct "thin outside fat inside (TOFI)" characteristic phenotype of obesity and type 2 diabetes compared to their Western counterparts. A recent genetics analysis on type 2 diabetes employing quantitative network models on a comprehensive database of genome-wide association studies (GWAS) catalog literature revealed the existence of different ancestry-specific human phenotype networks operating via disparate pathways in the pathogenesis of type 2 diabetes among Asians versus Westerners, thereby underscoring the importance of ancestry origins in the ultimate phenotype in this metabolic disorder [19].

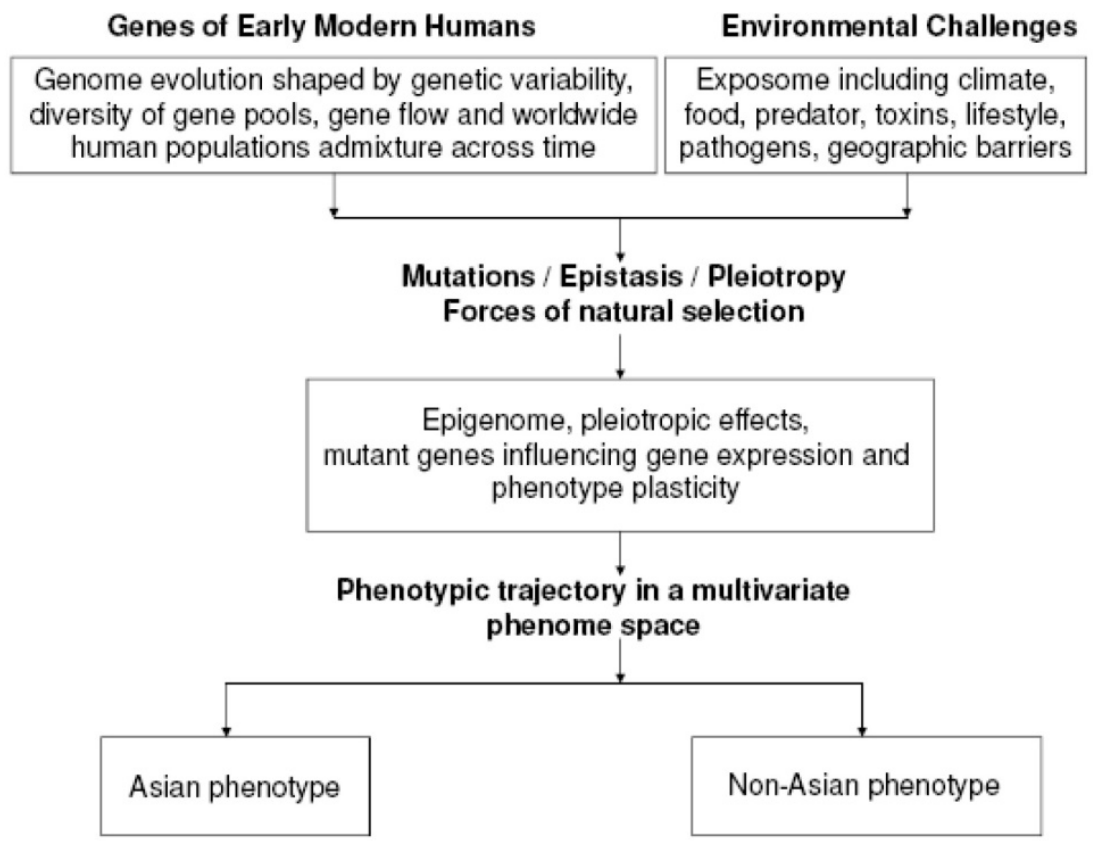

Figure 4. Hypothesis of the Origin of the Asian Phenotype. This illustrates a working model of the possible pathways of the genesis of the Asian phenotype from a multivariate phenome space based on intricate interactions between the genome and exposome. 


\section{Pharmacology}

\section{Warfarin metabolism}

Warfarin occurs as a racemic mixture of S- and R-enantiomers, with the former about 5 times more potent. Its narrow therapeutic index coupled with wide inter-individual dosing variations makes it notoriously challenging to adapt Western protocols for Asians. The differences in metabolism of S-warfarin via the cytochrome P450 (CYP) to 7-hydroxywarfarin, especially CYP 2C9, between Asians and Westerners are well-studied. Both CYP $2 \mathrm{C} 9 * 2$ and $2 \mathrm{C} 9 * 3$ alleles have a greater $\mathrm{Km}$ and lower Vmax for S-warfarin 7-hydroxylation than the CYP $2 \mathrm{C} 9 * 1$ allele [20]. Westerners possess a higher frequency of CYP $2 \mathrm{C}^{\star} 2(11 \%)$ which leads to a reduced activity of this enzyme by $30 \%$ compared with the 2C9*1 wild-type allele, and CYP 2C9*3 (7\%) which reduces the enzyme activity by $80 \%$. Asian Chinese and Malays however have not been found to harbor the CYP 2C9*2 allele while Asian Indians have this allele at a frequency of $4.35 \%$. The CYP2C $9 * 3$ allele is found in all the above three Asian ethnic groups, but at a lower frequency than the Westerners [21].

\section{Medical Oncology}

\section{Chemotherapy and cancer survival}

Rapid advances have transformed the oncology landscape ranging from screening, diagnostics, therapy, tumor responses, cytotoxic resistance and cancer prognosis. At all these fronts, evidence is accumulating that Asians vary significantly from Westerners. Molecular subtyping of breast cancer, for instance, revealed that estrogen receptor (ER) and progesterone receptor (PR) negative breast cancer are more prevalent among women from India and Pakistan, affecting younger women (below 40) compared to Westerners, with particularly aggressive triple negative or basal breast cancer (ER, PR, Her2/neu negative) subtype commoner in Asian Indians. A recent cross-sectional study also supported the finding that aggressive poorly-differentiated triple-negative breast cancers occur with greater frequency in young Asian women relative to Europeans [22]. Treatment efficacy also differs between Asians and non-Asians for a variety of reasons. Modern molecular therapies such as epidermal growth factor receptor (EGFR)-tyrosine kinase inhibitors for advanced non-small cell lung cancer have benefited more Asians than non-Asian patients related to the fact that EGFR mutations occur at higher frequency in Asians than non-Asians. In terms of survival, with the exception of South Asians, all other groups of Asian men (Chinese, Filipino, Japanese, Korean and Vietnamese) did better than Westerners with prostate cancer despite having paradoxically worse risk profiles such as older age and more advanced disease at diagnosis [23].

\section{Ophthalmology}

\section{Optic Neuritis}

A recent study showed that the incidence of optic neuritis in Singapore was 0.83 per 100,000, which was lower compared to reports from Sweden and Croatia but similar to Japan [24]. Among those affected in Singapore, the incidence was higher in the Chinese and Indians relative to the Malays. Pain at presentation occurred in $70.9 \%$ in a Singapore study compared to $92.2 \%$ to Westerners. This is similarly observed in Taiwan, Korea and Japan. Papillitis and disc hemorrhages were also far commoner among Asians compared to Western populations. Visual recovery rate is similar to Westerners though the recurrence rate is lower in Asians. The conversion rates of optic neuritis to multiple sclerosis among Asians seemed lower than those of Westerners [Optic Neuritis Study 25].

\section{Neurology}

\section{Stroke}

Asians have a higher prevalence of lacunar strokes and higher incidence among males of intracerebral hemorrhage [26]. There is also a relatively greater prevalence of strokes in younger patients and intracranial atherosclerosis. Yet, extracranial carotid atherosclerosis appears to be less common in Asia. Westerners in contrast more often suffer from cardioembolism, occluded carotid and vertebral arteries more than Asians. Asian Americans have more blockages involving deeper intracranial arteries than their Westerner counterparts. Subcortical ischaemic vascular dementia is more frequent in Asians because cerebral small vessel disease is commoner in Asians than Westerners [27]. Perhaps underlying genetic polymorphisms in homocysteine metabolism and differences in the pathways of thrombosis and fibrinolysis may explain the higher frequency of atherothromobotic stroke in Asians.

\section{Cardiology}

\section{Brugada syndrome}

The Brugada syndrome, characterized by incomplete right bundle branch block and ST elevations in anterior precordial leads on ECG is largely a voltage-gated $\mathrm{Na}_{\mathrm{v}} 1.5$ sodium channelopathy due to mutation of the SCN5A gene. Clinically, it is relatively asymptomatic till a cardiac catastrophe 
arises. Occasionally, life-threatening ventricular arrthythmias have been precipitated by fever, alcohol or drugs with sodium or calcium-channel blocking activity. Bezzina et al, identified a major Asian-specific haplotype SNP block not present among Westerners resulting in lower transcription activity of the SCN5A gene [28]. It is unknown if SCN5A promoter haplotype status influences the phenotype of Brugada syndrome among Asians or Westerners. Although not confined to Asians, the Brugada syndrome could be considered itself an Asian phenotype out of a spectrum of related cardiac electrophysiological channelopathies.

\section{Rheumatology}

\section{Systemic lupus erythematosus (SLE)}

A recent Canadian study of over a thousand SLE cases comprising 826 Westerners and 249 Asians found that Asian patients appeared to have the youngest age at onset with more frequent renal involvement and exposure to immunosuppressives compared with Western patients [29]. Chinese are more likely to suffer from arterial thromboembolic events whereas Westerners were more likely to have anti-phospholipid syndrome with venous thromboembolism. Another interesting study comparing SLE among community-based cohorts of Westerners and Chinese patients residing in Minnesota, USA and Singapore respectively showed that Asian patients have a greater tendency toward major organ involvement such as renal or cerebral disease compared with Westerners, which could contribute to the greater morbidity and mortality among Asians sufferers of SLE. Western patients were three times more likely than Asian patients to have serositis and seven times more likely than Chinese patients to have a hematologic complication. Also, Western SLE cases were far less likely to develop the characteristic malar rash of SLE compared to the Chinese patients [30]. More recently, single nucleotide polymorphisms of the protein tyrosine phosphatase non-receptor type 22 (PTPN22), a negative regulator of T-cell activation, was found to be different between European-Americans compared with Asians with respect to a population of over 8200 patients with different clinical sub-phenotypes of SLE, validating the notion of phenotypic variations of this disease between people according to their ancestral origins [31].

\section{Gastroenterology}

\section{Hepatitis C}

Data on treatment-naïve patients from a large multicenter study of combination ribavirin and peginterferon alfa-1a were analyzed by multivariate logistic regression and adjusted for these factors, and it was found that the Asian race was an independent predictor of achieving a sustained virological response compared to Westerners with chronic hepatitis C [32]. Furthermore, Westerners appear to have a greater propensity to hepatic fibrosis than Asians. Much attention has been focused on transforming growth factor beta-1 (TGF- $\beta 1$ ). The Arg25Pro SNP as well as proline on codon 10 of the TGF- $\beta 1$ gene confers an increased risk for progressive development of severe hepatic fibrosis among those with hepatitis $C$. These susceptibility allelic variants are significantly found among Westerners, and yet not among the Asian Chinese [33].

\section{Pulmonology}

\section{Obstructive Sleep Apnea}

Asians, in particular, seem to have a greater predilection for and also have much greater severity of this illness compared with Westerners matched for age, gender and BMI [34]. Based on objective polysomnographic data, Asian patients had significantly lower minimum oxygen saturation and higher esophageal pressures and respiratory disturbance index. Studies showed that for any given degree of OSA, Asians appeared to be less obese than Westerners; OSA occurs even among non-obese Asians [35]. OSA patients generally tended to have larger thyromental angles and higher Mallampati scores leading to a crowded posterior oropharynx couple with a steep thyromental plane than non-OSA patients. A Japanese study that focused on bony factors offered further clues. Compared to Western lateral cephalometric norms, Japanese people have smaller anteroposterior but proportionately greater vertical facial dimensions, with a dolico-facial pattern predisposing to OSA. This has obvious implications for companies developing and manufacturing home continuous positive airway pressure (CPAP) machines as they need to factor in these fundamental anatomical differences to adapt their mask devices to the Asian population.

\section{Nephrology}

\section{Diabetic Nephropathy}

It appears that the rate of involvement of the kidneys by diabetes mellitus is not only dependent on the glycemic control but also on racial factors [36]. In a large longitudinal cohort study, diabetic nephropathy was shown to be commoner in Asians than Westerners. Its progression to ESRD is also more likely among Asians than Westerners [37]. The prevailing hypothesis is that differences in functional 
renal reserve between Asians and Westerners may account for the phenotype encountered in Asians. Nitric oxide, which influences glomerular filtration, renal plasma flow and renal clearance of metabolites via its effects on renal vasodilatation, is an important determinant of the risk of diabetic nephropathy. Studies on nitric oxide in type 2 diabetes mellitus suggest that the renal reserve in Westerners is significantly higher than Asians and this is not confounded by duration of diabetes.

\section{Impact of the Asian Phenotype and Future Directions}

\section{Disease Burden and Management in Asia}

The Asian phenotype is a distinct 'effect modifier' and is essentially the collective 'phenome' arising from various systemic levels of biology unique to the Asian population (Table 1). Given the Asian phenotype's cross-cutting nature across many scientific fields and disciplines, the implications for medicine and public health are expectedly profound. Variations from the perceived 'standard' could be small and irrelevant at times and yet prove crucial with devastating consequences if ignored at other times. As the Asian phenotype gets factored into the diagnostic and therapeutic equation, differing cutoffs and thresholds will imply that disease burden and healthcare costs could change significantly. The end result can affect the intensity of health screening efforts, medical expenditure and re-shape public health policies.

\section{Medical Research and Clinical Trials on Asians}

Biopharmaceutical industries have largely progressed ahead to include Asians in most of their clinical trials to ensure that their conclusions and drug indications are equally applicable in the setting of non-Asians and Asians alike to facilitate the registration of their products in countries with strict regulatory authorities governing the use of pharmaceutical agents and medical devices in their unique populations. Asian countries could impose on drug companies to repeat studies that have been conducted only on non-Asians so as to demonstrate either equivalence or any significant differences for any agent before it is deemed safe for their population.

\section{Implications for Personalized Medicine in Asia}

The post-genomic era brings the model of "individualized therapy" a step closer to reality. This so-called " $\mathrm{N}=1$ clinical trial" enables physicians to prescribe the "right treatments to the right patient at the right time" with the least amount of guesswork in terms of efficacy and safety. Personalized and precision medicine, though still in its infancy, is poised to mature and expected to become a standard process at the bedside in the near future. As personalized medicine morphs into the accepted norm, the Asian phenotype will be dissected by "-omics" technologies and systems biology to allow more precise prediction of treatment responses within any given Asian individual.

\section{Limitations}

Variation of a trait between individuals within a race has been reported to be much greater in magnitude than the mean variation between races though this finding remains disputable. This may apply to multifactorial traits. In such instances, the restriction of the study population to a particular race or ethnic group would clearly limit the effectiveness of the study. In the future, specific human pharmacogenetic phenotypes or phenomes might become extra biological factors to be considered in addition to such subdivisions into an Asian or Caucasian phenotype.

Table 1. Asian Phenotypes Recognizable at Various System Levels of Organization

\begin{tabular}{|c|c|}
\hline LEVEL OF & EXAMPLES OF ASIAN PHENOTYPE \\
\hline Molecular / & VKORC1 low warfarin dose haplotype \\
\hline Subcellular & $\begin{array}{l}\text { PML gene breakpoint cluster region- } 1 \text { subtype } \\
\text { (bcr1) of chromosomal translocation } \mathrm{t}(15: 17) \text { is } \\
\text { more frequent in Asians than Westerners }\end{array}$ \\
\hline \multirow[t]{2}{*}{ Cellular / Tissue } & $\begin{array}{l}\text { Cartilage chondrocalcinosis rarer in Asians than } \\
\text { among Westerners }\end{array}$ \\
\hline & $\begin{array}{l}\text { Nasopharyngeal cancer (NPC) predominantly } \\
\text { differentiated non-keratinizing carcinoma (WHO } \\
\text { Type } 2 \text { histology) among Chinese whereas NPC in } \\
\text { Westerners largely keratinizing subtype (WHO } \\
\text { Type 1) }\end{array}$ \\
\hline \multirow[t]{2}{*}{ Organ / Systemic } & $\begin{array}{l}\text { 1. Mean axial length of eyeball highest in East } \\
\text { Asians associated with the most myopic mean } \\
\text { spherical equivalent whereas Westerners have the } \\
\text { most hyperopic mean spherical equivalent. }\end{array}$ \\
\hline & $\begin{array}{l}\text { Cardiac sodium channelopathy from SCN5A } \\
\text { loss-of-function mutation with arrthymogenic } \\
\text { susceptibility, sudden death (Brugada syndrome) }\end{array}$ \\
\hline \multirow[t]{2}{*}{$\begin{array}{l}\text { Organismal / } \\
\text { Population }\end{array}$} & $\begin{array}{l}\text { Metabolic syndrome diagnosed at lower } \\
\text { anthropometric cutoffs of waist circumference } \\
\text { (visceral adiposity) }\end{array}$ \\
\hline & $\begin{array}{l}\text { Insulin resistance (whole body and } \\
\text { insulin-sensitive specific organs of individuals } \\
\text { and entire human population) higher among } \\
\text { certain ethnic groups of Asians such as Indians } \\
\text { compared with Westerners }\end{array}$ \\
\hline
\end{tabular}

\section{Summary and Conclusions}

There is an increasing recognition of atypical, yet distinctive, features of many diseases seen in large population segments across Asia that may be termed the 'Asian phenotype'. This Asian phenotype applies across many different disciplines in Medicine. More 
emphasis should be placed on studying its impact on diagnosis, treatment and clinical research particularly as our understanding of the connections between the genome, epigenome, epitranscriptome and ultimately the phenome broadens with technological advances.

\section{Acknowledgements}

The patient editorial assistance offered by Jane Sim-Joo Tan in the preparation of this manuscript is deeply appreciated.

\section{Competing Interests}

The author has declared that no competing interest exists.

\section{References}

1. Tabata Y AC. Globalising clinical development in Japan. . J Commercial Biotechnol 2008; 14: 73-8.

2. bureau Pr. World population data sheet. 2008

3. NLM. Other Notable MeSH Changes and Related Impact on Searching: Ethnic Groups and Geographic Origins. NLM Technical Bulletin 2003; 335.

4. Deurenberg P, Deurenberg Yap M, Wang J et al. The impact of body build on the relationship between body mass index and percent body fat. International journal of obesity and related metabolic disorders : journal of the International Association for the Study of Obesity 1999; 23: 537-42.

5. Zhang YY, Liu PY, Lu Y et al. Race and sex differences and contribution of height: a study on bone size in healthy Caucasians and Chinese. American journal of human biology : the official journal of the Human Biology Council 2005; 17: $568-75$

6. Okada Y, Kamatani Y, Takahashi A et al. A genome-wide association study in 19633 Japanese subjects identified LHX3-QSOX2 and IGF1 as adult height loci. Human molecular genetics 2010; 19: 2303-12.

7. Nightingale CM, Rudnicka AR, Owen CG et al. Patterns of body size and adiposity among UK children of South Asian, black African-Caribbean and white European origin: Child Heart And health Study in England (CHASE Study). International journal of epidemiolooy 2011; 40: 33-44.

8. Excoffier L, Smouse PE, Quattro JM. Analysis of molecular variance inferred from metric distances among DNA haplotypes: application to human mitochondrial DNA restriction data. Genetics 1992; 131: 479-91.

9. Karlow W BL. Interethnic factors affecting drug response. . Adv Drug Res 1994; 23: 53.

10. Cann RL, Stoneking M, Wilson AC. Mitochondrial DNA and human evolution. Nature 1987; 325: 31-6.

11. Campbell MC, Tishkoff SA. African genetic diversity: implications for human demographic history, modern human origins, and complex disease mapping. Annual review of genomics and human genetics 2008; 9: 403-33.

12. Nei M, Roychoudhury AK. Evolutionary relationships of human populations on a global scale. Molecular biology and evolution 1993; 10: 927-43.

13. Cooper GM, Nickerson DA, Eichler EE. Mutational and selective effects on copy-number variants in the human genome. Nature genetics 2007; 39: S22-9.

14. Pigliucci M. Developmental phenotypic plasticity: where internal programming meets the external environment. Current opinion in plant biology 1998; 1: 87-91.

15. Tan CE, Ma S, Wai D et al. Can we apply the National Cholesterol Education Program Adult Treatment Panel definition of the metabolic syndrome to Asians? Diabetes care 2004; 27: 1182-6.

16. Misra A, Vikram NK. Insulin resistance syndrome (metabolic syndrome) and obesity in Asian Indians: evidence and implications. Nutrition 2004; 20: 482-91.

17. Lim LL, Tan AT, Moses $\mathrm{K}$ et al. Place of sodium-glucose cotransporter-2 inhibitors in East Asian subjects with type 2 diabetes mellitus: Insights into the management of Asian phenotype. Journal of diabetes and its complications 2016.

18. Karamali NS, Ariens GA, Kanhai $\mathrm{HH}$ et al. Thin-fat insulin-resistant phenotype also present in South Asian neonates born in the Netherlands. Journal of developmental origins of health and disease 2015; 6: 47-52.

19. Qiu J, Moore JH, Darabos C. Studying the Genetics of Complex Disease With Ancestry-Specific Human Phenotype Networks: The Case of Type 2 Diabetes in East Asian Populations. Genetic epidemiology 2016; 40: 293-303.

20. Aithal GP, Day CP, Kesteven PJ, Daly AK. Association of polymorphisms in the cytochrome P450 CYP2C9 with warfarin dose requirement and risk of bleeding complications. Lancet 1999; 353: 717-9.

21. Gage BF, Lesko LJ. Pharmacogenetics of warfarin: regulatory, scientific, and clinical issues. Journal of thrombosis and thrombolysis 2008; 25: 45-51.

22. Tea MK, Fan L, Delancey JW et al. Is breast cancer in young Asian women more aggressive than in Caucasians? A cross-sectional analysis. Tumour biology : the journal of the International Society for Oncodevelopmental Biology and Medicine 2013; 34: 2379-82.
23. Robbins AS, Yin D, Parikh-Patel A. Differences in prognostic factors and survival among White men and Black men with prostate cancer, California, 1995-2004. American journal of epidemiology 2007; 166: 71-8.

24. Lim SA, Wong WL, Fu E et al. The incidence of neuro-ophthalmic diseases in Singapore: a prospective study in public hospitals. Ophthalmic epidemiology 2009; 16: 65-73.

25. Group ONS. The clinical profile of optic neuritis. Experience of the Optic Neuritis Treatment Trial. Optic Neuritis Study Group. Arch Ophthalmol 1991; 109: 1673-8.

26. Ng WK GK, George J, Tan CT, Baird A, Donnan A. A comparison study of stroke subtypes between Asian and Caucasians in two hospital based stroke registries. . Neurol J Southeast Asia 1998 1998; 3: 19-26.

27. Sen S, Dahlberg K, Case A et al. Racial-ethnic differences in stroke risk factors and subtypes: results of a prospective hospital-based registry. The International journal of neuroscience 2013; 123: 568-74

28. Bezzina CR, Shimizu W, Yang P et al. Common sodium channel promoter haplotype in asian subjects underlies variability in cardiac conduction. Circulation 2006; 113: 338-44.

29. Peschken CA, Katz SJ, Silverman E et al. The 1000 Canadian faces of lupus: determinants of disease outcome in a large multiethnic cohort. The Journal of rheumatology 2009: 36: 1200-8.

30. Thumboo J, Uramoto K, O'Fallon WM et al. A comparative study of the clinical manifestations of systemic lupus erythematosus in Caucasians in Rochester, Minnesota, and Chinese in Singapore, from 1980 to 1992. Arthritis and rheumatism 2001; 45: 494-500.

31. Namjou B, Kim-Howard X, Sun C et al. PTPN22 association in systemic lupus erythematosus (SLE) with respect to individual ancestry and clinical sub-phenotypes. PloS one 2013; 8: e69404.

32. Missiha S, Heathcote J, Arenovich T, Khan K. Impact of asian race on response to combination therapy with peginterferon alfa-2a and ribavirin in chronic hepatitis C. The American journal of gastroenterology 2007; 102: 2181-8.

33. Wang H, Mengsteab S, Tag CG et al. Transforming growth factor-beta1 gene polymorphisms are associated with progression of liver fibrosis in Caucasians with chronic hepatitis C infection. World journal of gastroenterology : WJG 2005; 11: 1929-36.

34. Ong KC, Clerk AA. Comparison of the severity of sleep-disordered breathing in Asian and Caucasian patients seen at a sleep disorders center. Respiratory medicine 1998; 92: 843-8.

35. Li KK, Kushida C, Powell NB et al. Obstructive sleep apnea syndrome: a comparison between Far-East Asian and white men. The Laryngoscope 2000; 110: 1689-93.

36. Earle KK, Porter KA, Ostberg J, Yudkin JS. Variation in the progression of diabetic nephropathy according to racial origin. Nephrology, dialysis, transplantation : official publication of the European Dialysis and Transplant Association - European Renal Association 2001; 16: 286-90.

37. Young BA, Maynard C, Boyko EJ. Racial differences in diabetic nephropathy, cardiovascular disease, and mortality in a national population of veterans. Diabetes care 2003; $26: 2392-9$. 